\title{
Improved Stall Delay Model for HAWT Performance Predictions using 3D Navier-Stokes Solver and Actuator Disk Method
}

\author{
M. N. Hamlaoui ${ }^{1}$, A. Smaili ${ }^{1 \dagger}$ and H. Fellouah ${ }^{2}$ \\ ${ }^{1}$ Laboratory of Mechanical Engineering and Development (LGMD), National Polytechnic School -ENP-, \\ P.B. 182 El-Harrach, Algiers, 16200, Algeria \\ ${ }^{2}$ Department of Mechanical Engineering, University of Sherbrooke, Quebec, Canada \\ †Corresponding Author Email: arezki.smaili@g.enp.edu.dz
}

(Received February 1, 2021; accepted July 20, 2021)

\begin{abstract}
The Actuator Disk Method (ADM), in its analytical formulation or combined with Navier-Stokes equations, is widely used for design and/or for aerodynamic analysis of Horizontal Axis Wind Turbines (HAWT). This method has demonstrated its capabilities for performance predictions of HAWT rotors for limited range of wind speeds with lower angles of attack values, i.e. attached flow conditions. However, for typical wind speeds that rotor encounters, under higher angles of attack i.e. stall conditions, such a method cannot describe accurately the flow characteristics around rotor-blades due to severe flow separations coupled with the effects of blades rotation as well as the radial flow over the blades. In this paper, original correction approaches have been proposed for the existing stall delay models to take into account both the blade rotation and the radial flow effects over the rotor blades. For this purpose, the ADM combined with 3D- NavierStokes equations formulation using Large Eddy Simulations (LES) model has been considered to describe the incompressible turbulent flow field around HAWT rotor blades. The resulting mathematical model has been solved using a 3D in-house subroutine developed with OpenFOAM code. The proposed numerical method has been validated against the well recognized reference measurements obtained using the New MEXICO and the NREL Phase VI experimental wind turbines. In comparison with existing stall delay models, the proposed correction approaches, especially the radial flow approach, have shown noticeable enhancements on performance predictions of HAWT rotors compared to the experimental measurements. It has been found very low discrepancies to the experimental torque and thrust values, up to $1 \%$ and $10 \%$ have been recorded respectively.
\end{abstract}

Keywords: Actuator disk method; HAWT, Aerodynamic performances; Stall delay model; LES model; Numerical simulation.

NOMENCLATURE

\begin{tabular}{|c|c|c|c|}
\hline$B$ & rotor number of blades & $U_{\text {rel }}$ & $\begin{array}{l}\text { flow velocity relative to blade local } \\
\text { station }\end{array}$ \\
\hline$r$ & local radial station & $U_{n}$ & $\begin{array}{l}\text { normal component of the flow } \\
\text { velocity relative to blade local station }\end{array}$ \\
\hline$c$ & local chord at given radial station & $U_{t}$ & $\begin{array}{l}\text { tangential component of the flow } \\
\text { velocity relative to blade local station }\end{array}$ \\
\hline$U_{\infty}$ & free stream velocity & $f_{z}$ & axial volume force component \\
\hline$T S R, \lambda$ & tip speed ratio & $f_{\theta}$ & tangential volume force component \\
\hline$C_{l}, C_{d}$ & corrected lift and drag coeffecients & $f_{r}$ & radial volume force component \\
\hline$C_{l, \exp }$ & measured lift coefficient & $\gamma$ & coning angle \\
\hline$C_{l, i n v}$ & inviscid lift coefficient & $\varphi$ & flow angle \\
\hline$f_{l}, f_{d}$ & $\begin{array}{l}\text { separation factors for the lift and the drag } \\
\text { coefficients variation }\end{array}$ & $\beta$ & twist angle \\
\hline$C_{n}, C_{t}$ & normal and tangential force coefficients & $\theta_{p}$ & pitch angle \\
\hline AoA, $\alpha$ & angle of attack & $\Omega$ & rotational speed \\
\hline
\end{tabular}


M. N. Hamlaoui et al. / JAFM, Vol. 15, No. 1, pp. 37-50, 2022.

\section{INTRODUCTION}

With the technological and industrial development attained in the last decades in order to satisfy human needs, the world has seen a significant increase of the energy request. Most of the energy sources are mainly of fossil origin due to their cheapness, effectiveness and storage easiness. Nevertheless, fossil fuels are non-renewable resources emitting large amounts of carbon dioxide, once brunt, which leads to the appearance of greenhouse effect, negative environment changes and fatal diseases among people. According to the International Renewable Energy Agency (IRENA (2019)), transformation towards cleaner and renewable energy resources has been noticed where the projected electricity generation, based on renewables, will reach $86 \%$ by 2050 , a third of which is wind power. The rapid drop of wind energy cost has provided motivation for manufacturers and researchers to conduct several research on optimization and performance predictions of Horizontal Axis Wind Turbines (HAWT) (Hansen 2008). For HAWT optimization and performance predictions, the actuator disk concept, used for the first time by Rankine (1865) and Froude (1889), is one of the widely used approaches. According to Horlock (1978), it consists of permeable surface, instead of the real geometry where the external forces provided by the rotor act upon the incoming flow causing pressure jump and strong velocity field gradients. The actuator disk is a mathematical model of an ideal rotor rooted on four main assumptions; the fluid flow accross the control volume is considered ideal (inviscid, incomprissible, irrotational with no turbulence effect), an infnite number of blades is considred, the flow is uniform and unidimensional accross the disk surface and the static pressure far upstream and downstream of the rotor is assumed undisturbed. Tip loss correction approaches have been included in order to correct the infinite number of blades assumption i.e. Shen et al. (2005), Wimshurst and Willden (2017). The computational application of the actuator disk concept has been used in its analytical approaches such as the blade element momentum method (Glauert 1963; Tahani and Moradi 2016) or with the combination of the blade element theory with the Navier-Stokes equations due to the development of Computational Fluid Dynamics (CFD) methods and the High Performance Computing (HPC) tools. Sørensen and Myken (1992) have been the first to use the actuator disk concept where the blade forces are computed through the blade element approach combined with the airfoil aerodynamic properties. Masson et al. (2000), Masson et al. (2001) used the ADM to investigate first the tower-shadow impacts on HAWT and then studied the unsteady flow phenomena around the rotor. Based on the last approach, later, Smaïli and Masson (2003), Smaïli and Masson (2004), Masson and Smailli (2006) works on the nacelle anemometry of typical HAWT rotors have been reported. Mainly, they tackled the impact of rotor aerodynamic key parameters upon the flow features within the nacelle region, especially, around the anemometer location of $600 \mathrm{~kW}$ commercially available HAWT at a distance of $5.7 \mathrm{~m}$. Tata et al. (2018) also performed a simulation study on nacelle anemometer using ADM approach, with the emphasis on the effect of grid topology upon numerical predictions accuracy. Wind farm large eddy simulations have been conducted by Stevens et al. (2018) where comparison using the Actuator Disk Method (ADM) and the Actuator Line Method (ALM) with wind tunnel experiments have been carried out. A total number of 1.76 million nodes for the ADM and ALM calculations has been used with a time step for the ADM calculation 100 times greater than the one used for ALM. They concluded that the ADM provides good wake predictions with lower mesh requirements and computational costs compared to the ALM. Recently, Sørensen et al. (2020) proposed an analytical model for representing HAWT rotor body forces for numerical ADM computations on wind farms based on the assumption that HAWT rotor disk is subject to a constant circulation modified for tip and root effects; it has been generalized and depends only on the knowledge of the HAWT rated wind speed and the nameplate capacity. It has been validated with the 2MW Tjaereborg and the 10MW DTU HAWT where good agreement has been recorded for two tested wind turbines.

The strongest of hybrid methods i.e. ADM consists on their reliability and capability for performance and wake predictions of HAWT rotors for specific range of flows corresponding to an attached flow regimes because during the $\mathrm{ADM}$ calculation process, the airfoil characteristics used for the sectional forces estimation are obtained from wind tunnel experiments for a fixed blade (non-rotating blade). Nevertheless, for typical incoming wind speeds stall occurs (stall conditions) and the effect of rotation appears which leads to performances to be under-predicted and such method breakdown. Himmelskamp (1947) has been the first to record, through a qualitative analysis on propeller blade, the increase of the loads in rotational case, he postulated that this augmentation is due to the centrifugal and Coriolis forces. On the other hand, it has been shown by McCroskey (1971) that the centrifugal forces acting on the separated flow region move the flow mass from the root of the blade toward the tip describing the process as a centrifugal pumping. Madsen and Rasmussen_(1988) suggest that the appeared radial forces on the separated regions tends to create radial pressure gradient which produce a radial flow. Hu et al. (2006) conducted full CFD and experimental study on HAWT's stall delay (effect of rotation) where they concluded that Coriolis and centrifugal forces are strong on the near root region of the rotor blade at stall conditions which act as favorable pressure gradient tending to delay separation occurrence. Several attempts have been done to describe the phenomenon by proposing correction models based on different assumptions. Snel et al. (1994), Lindenburg (2004), Dumitrescu and Cardos (2012) proposed simple models by assuming that the effect of rotation causes the lift coefficient to increase without any variation for the drag coefficients. Du and Selig (1998), Chaviaropoulos and Hansen (2000) proposed corrections for both lift and drag coefficients to take 
into account the 3D effect. Breton et al. (2008) have studied six different Stall Delay Models (SDM) available on literature through the NREL Phase VI wind turbines measured torque, thrust and the blade sectional forces where significant over-estimations and important discrepancies have been recorded; they concluded that all models are unable to represent correctly the effect of rotation phenomenon due to the lack of generality. Yu et al. (2011) confirmed later, through comparison between 3D airfoil data extracted from full CFD simulations and the corrected lift and drag coefficients using the existing SDM, that the presented models are unable to predict correctly the effect of rotation. Recently, Amini et al. (2021) proposed modifications for Snel et al. (1994) SDM empirical coefficients than studied the effect of the 3D airfoil corrections on the MEXICO rotor measured blade sectional forces and near wake upstream and downstream from the rotor, for an attached and separated flow conditions, using the ADM implemented in OpenFOAM where a certain improvement of predicted loads has been noticed. Nevertheless, important discrepancies are still recorded for the loads prediction.

The literature shows clearly the lack of existing stall delay models to predict accurately the performances of HAWT rotor particularly at higher angles of attack, i.e. stall conditions, due to severe flow separations coupled with the effects of blades rotation as well as the radial flow over the blades. In this paper, original correction approaches have been proposed to take into account both the blade rotation and the radial flow effects over the rotor blades,

in order to improve the accuracy of the existing stall delay model predictions. For this purpose, the wind turbine rotor is modeled by the ADM and the flowfield is described by 3D Navier-Stokes equations using LES model. The resulting mathematical model is implemented within OpenFOAM code. The validity of the proposed numerical method has been carried out through New MEXICO (Schepers et al. (2014), Boorsma and Schepers (2016)) and the NREL Phase VI (Simms et al. (2001), Fingersh et al. (2001)) experimental wind turbines. The capability of the proposed numerical method to predict consistent results is presented and discussed.

\section{MATHEMATICAL MODEL}

\subsection{Actuator Disk Method (ADM)}

The ADM consists in rotor modeling by combining the actuator disk concept and the blade element theory. These are described as follows.

Actuator disk concept: It consists in modeling the rotor using body forces. A permeable surface, instead of the actual rotor geometry, defined by the rotor swept area on which the forces associated to the turbine blades, defined as surficial forces, acts upon the incoming flow (Masson et al. (2001)). For HAWT, Fig. 1 shows that the actuator disk geometry, defined by the rotor swept area $(A)$, is a circular cone with a base radius of $(R \cos (\gamma))$, where $\gamma$ is the

coning angle and $\mathrm{R}$ is the rotor blade length. As the blade element theory has been used to determine the wind turbine blades force, the acting surficial force exerted by an elementary actuator disk surface $d A$ could be divided into tangential and normal component denoted, respectively, $d F_{\theta}$ and $d F_{z}$ with no radial effect on the flow field $\left(d F_{r}=0\right)$.

Blade element theory: It has been often applied to determine the required body force. The rotor is composed of $B$ number of blades of length $R$ and a pitch angle $\theta_{p}$. Figure 2 (a) shows the rotor blade at a certain azimuthal position with a rotational speed $\Omega$ defined on a cylindrical reference frame $(\vec{\theta}, \vec{z}, \vec{r})$ where $\vec{\theta}, \vec{z}$ and $\overrightarrow{\mathrm{r}}$ are, respectively, the tangential, axial and radial directions. A cross section A-A' of the blade for a given radius $r$, shown by Fig. 2 (b), defines the developed forces over the blade section of a chord length $\mathrm{c}$ and twist angle $\beta$ due to lift and drag forces. The flow velocity relative to the local blade station $\left(U_{r e l}\right)$ can be decomposed, in the cross sectional element of airfoil plane, into normal and tangential components $\left(U_{n}, U_{t}\right)$ defined as follows:

$U_{\text {rel }}=\sqrt{U_{n}^{2}+U_{t}^{2}}$

where $U_{\mathrm{n}}=-\mathrm{u}_{\mathrm{k}} \mathrm{z}_{\mathrm{k}}$

and $\quad \mathrm{U}_{\mathrm{t}}=\left(\Omega \mathrm{r}-\mathrm{u}_{\mathrm{k}} \theta_{\mathrm{k}}\right)$

$u_{k}$ is the $k^{\text {th }}$ velocity component, where $\mathrm{z}_{\mathrm{k}}$ and $\theta_{k}$ are the cosine directors of the unit vectors $\mathrm{z}$ and $\theta$.

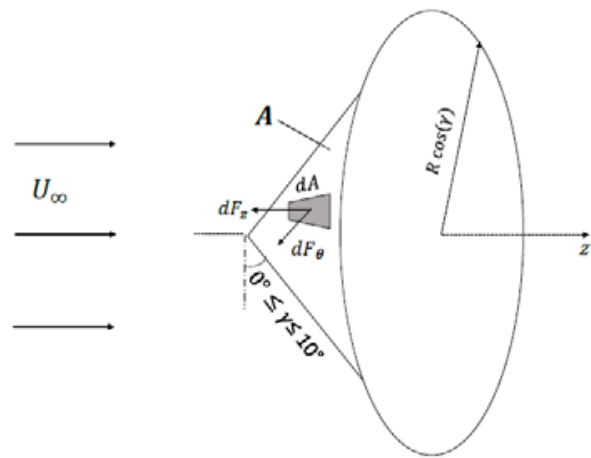

Fig. 1. Actuator disk concept.

Table 1. Proposed coefficients for the functions $g_{i} ; g_{a x}$ and $g_{t a n}$ for both axial and tangential forces respectively.

\begin{tabular}{|c|c|c|c|}
\hline & \multicolumn{2}{|c|}{$\mathrm{g}_{\mathrm{ax}}$} & \multicolumn{2}{c|}{$\mathrm{g}_{\tan }$} \\
\hline $\mathrm{a}_{\mathrm{ax}}$ & $\mathrm{b}_{\mathrm{ax}}$ & $\mathrm{a}_{\tan }$ & $\mathrm{b}_{\tan }$ \\
\hline 0.1219 & 21.52 & 0.0984 & 13.026 \\
\hline
\end{tabular}

The geometric angle of attack $(\alpha)$ could be defined as follows:

$\alpha=\arctan \left(\frac{U_{n}}{U_{t}}\right)-\left(\beta+\theta_{p}\right)$ 
(a)

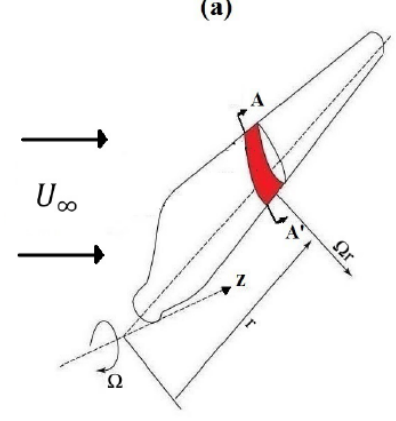

(b)

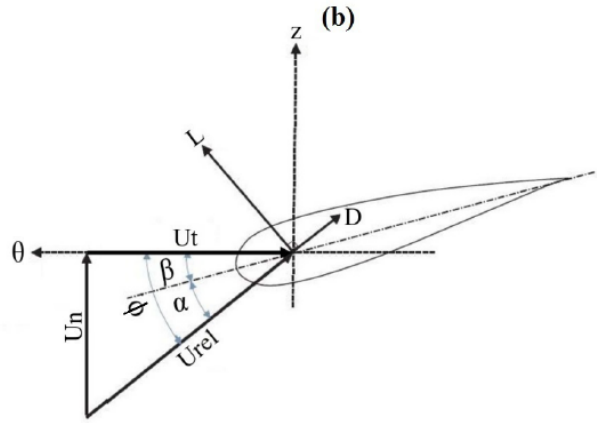

Fig. 2. (a) Rotor blade for a given azimuthal position (b) Cross section A-A' at a given radial station.

Several tip loss correction models have been proposed to correct the actuator disk infinite number of blades assumption (Prandtl and Betz 1927; Glauert 1963; Shen et al. 2005; Wimshurst and Willden 2017). Furthermore, to take into account the effects of the blade tip vortices, the airfoil characteristics have to be corrected using the recently proposed Wimshurst and Willden (2017) tip-loss factor. It consists on calibration of Shen et al. (2005) tip loss correction model where the tangential and axial forces are corrected separately as expressed by Eq. 5, Eq. 6 and Table 1.

$\mathrm{F}=\frac{2}{\pi} \cos ^{-1}\left[\exp \left(-\mathrm{g}_{\mathrm{i}} \frac{\mathrm{B}\left(1-\frac{\mathrm{r}}{\mathrm{R}}\right)}{2 \frac{\mathrm{r}}{\mathrm{R}} \sin \phi}\right)\right]$

where

$g_{i}=\exp \left(-a_{i}\left(B \lambda-b_{i}\right)\right)+0.1$

Where $\mathrm{i}$ indicates the axial and tangential forces direction. The forces should be calculated and introduced in the momentum equations as forces per unit volume of the blade length. For an infinitesimal volume at blade section, the resulting axial and tangential volume forces are given as follows:

$f_{\mathrm{z}}=\frac{\mathrm{dF}_{\mathrm{z}}}{\mathrm{dv}}=\frac{1}{4 \pi \mathrm{rdz}} \mathrm{B} \rho \mathrm{U}_{\mathrm{rel}}^{2} \mathrm{FC}_{\mathrm{n}} \mathrm{c}$

$f_{\theta}=\frac{\mathrm{dF}_{\theta}}{\mathrm{dv}}=\frac{1}{4 \pi \mathrm{rdz}} \mathrm{B} \rho \mathrm{U}_{\mathrm{rel}}^{2} \mathrm{FC}_{\mathrm{t}} \mathrm{c}$

For the ADM theory, the radial volume force component is considered to be zero $\left(f_{r}=0\right)$.

\subsection{Existing Stall Delay Models (SDM)}

The stall delay effect is still an inapprehensible phenomenon, several researchers tried to model and to explain this problem with different approaches by proposing several solutions based on different assumptions. Some models were based on theoretical analyses (Du and Selig 1998; Lindenburg 2004) while the other models were developed empirically from experimental measurements (Dumitrescu et al. 2007; Corrigan and Schillings 1994). The most used and wellknown, Du and Selig (1998), SDM has been used and expressed as follows:

$$
\begin{aligned}
& \mathrm{C}_{\mathrm{l}}=\mathrm{C}_{1,2 \mathrm{~d}}+\mathrm{f}_{\mathrm{l}} \Delta \mathrm{C}_{\mathrm{l}} \\
& \mathrm{C}_{\mathrm{d}}=\mathrm{C}_{\mathrm{d}, 2 \mathrm{~d}}+\mathrm{f}_{\mathrm{d}} \Delta \mathrm{C}_{\mathrm{d}}
\end{aligned}
$$

where the specific functions $f_{l}$ and $f_{d}$ represent, respectively, the variation of the lift and the drag coefficients. $\Delta C_{l}=C_{l, \text { inviscid }}-C_{l, 2 d}$. The inviscid lift coefficient $C_{l, \text { inviscid }}=2 \pi\left(\alpha-\alpha_{0}\right), \alpha_{0}$ is the Angle of Attack (AoA) at the zero lift coefficient and $C_{l, 2 d}$ is the $2 \mathrm{D}$ lift coefficient. $\Delta C_{d}=C_{d, 2 d}-$ $C_{d, 0}$ is the difference between the $2 \mathrm{D}$ drag coefficient and the drag coefficient at zero AoA.

\subsection{Proposed improvement approaches for Stall delay model}

As mentioned above, at higher AoAs, i.e. stall conditions, accurate predictions of aerodynamic characteristics and loads for a typical rotor blade require that the $2 \mathrm{D}$ lift and drag coefficients $\left(C_{l, 2 d}\right.$ and $C_{d, 2 d}$ ), used during the ADM calculation, to be corrected to take into account the effect of rotation and the radial flow effect.

\subsubsection{Improved Stall Delay Model with new rotation correction approach (ISDM- \\ Rot)}

Figure 3 shows that the experimental 3D lift coefficient of a rotating blade has a specific and a non linear evolution versus the AoA. As it can be seen, it increases with the increase of the AoA to attain the maximum at an AoA much higher than the 2D one (estimated as (Snel et al. 1994; Du and Selig 1998; Lindenburg 2004)); once this angle is exceeded, the 3D experimental lift coefficient decreases which could explained as the stall occurrence. This could be explained as the effect of rotation tends to delay the boundary layer separation to specific $A o A$ (denoted $\alpha_{s}$ ) higher than the AoAattained in stationary case, once $\alpha_{S}$ has been exceeded stall occurs and provide performances drop. Physically explained, the linear evolution at the near root region of the rotor blade obtained using existing SDM correction methodology tends to delay stall to infinity which causes loads over predictions for high AoA (high incoming wind speeds). To take into 


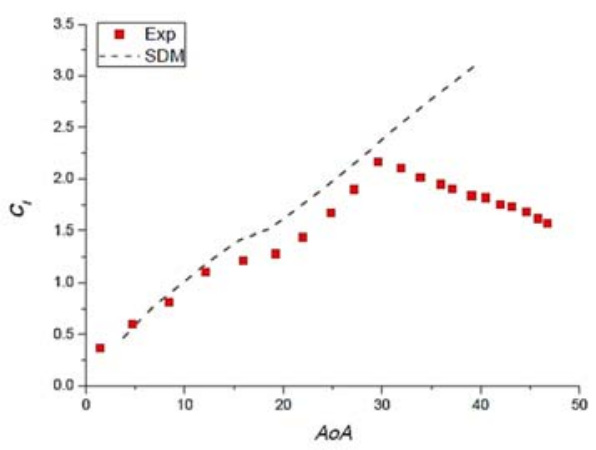

Fig. 3. Predicted 3D lift coefficient for the NREL Phase VI rotor at $r=R=0: 30$ span wise position using the existing stall delay models.

account the SDM limitation, a new correction approach for the lift coefficient has been proposed in this study. It is based on comparing the $2 \mathrm{D}$ lift coefficient to the 3D lift coefficient measured on the NREL Phase VI rotor for different radial positions. Figure 4 shows the resulting evolutions of the shift parameter $f_{s}$, defined in Eq. (11), obtained for the NREL Phase VI rotor. As it can be seen, there are three different behaviors along the rotor blade:

$\mathrm{f}_{\mathrm{s}}=\frac{\mathrm{C}_{1, \exp }-\mathrm{C}_{1,2 \mathrm{~d}}}{\mathrm{C}_{1,2 \mathrm{~d}}}$

- In the near root region $(r / R \leq 0.3)$, the shift parameter is close to $0(0 \%)$ for AoAs under $12^{\circ}$, which means that there is no effect of rotation. Nevertheless, for AoAs upper than $12^{\circ}$, the shift parameter increases with the increase of the AoAto attain a maximum value of 1.5 (rotational augmentation of $150 \%$ ) at an $\mathrm{AoA}$ of $33^{\circ}$, it decreases then for AoAs upper than $33^{\circ}$.

- In the near tip region $(r / R \geq 0.95)$, the shift parameter shows a negative shape that is due to the tip loss phenomenon. For this reason, the variation in this region is not considered in the present study.

- In the middle blade region $(0.3<r / R<$ 0.95), the shift parameter tends to have a $2 \mathrm{D}$ behavior for AoAs under $12^{\circ}$, it increases with the increase of the AoA to maximum value around the AoA of $22^{\circ}$ and decreases with the increase of the AoAs to achieve the $2 \mathrm{D}$ behavior around the AoA of $34^{\circ}$.

These results show that the effect of rotation is significant on the near root region of the rotor blade $(r / R \leq 0.30)$ and as the evolutions of the shift parameter are similar in the middle blade region, the idea consists on averaging them. In the present study, a new formulation for the shift parameter is proposed below for the two regions; near root and middle blade:

$f_{s}=a \exp \left(-\left(\frac{\alpha-\alpha_{s}}{d}\right)^{2}\right)$

Where ${ }_{\mathrm{a}}, \alpha_{s}$ and $\mathrm{d}_{\text {are constants which represent the }}$ amplitude, the specific AoA that corresponds to the

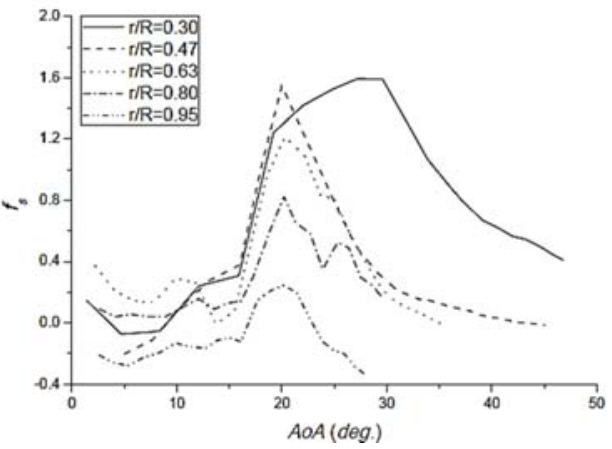

Fig. 4. Empirical evolution of the shift parameter as a function of AoA obtained at different radial positions for the NREL Phase VI blade.

peak (delayed stall angle) and the controlling peak width respectively. The constants $a, \alpha_{s}$ and $d$ appropriate to the model have been calibrated based on the NREL Phase VI lift coefficient evolutions and are equal, respectively, to $1.45,0.575\left(33^{\circ}\right), 0.28$ $\left(16^{\circ}\right)$ for radial positions $r / R \leq 0.30$ and $0.55,0.38$ $\left(22^{\circ}\right), 0.12\left(6.88^{\circ}\right)$ for radial positions $r / R>0.30$.

Validation of the new lift coefficient correction approach will be discussed in Sec.4.

\subsubsection{Radial flow correction}

The effect of rotation is due to the appearance of radial flow over the rotor blade where as the flow condition is more deeply separated, it becomes important (Himmelskamp 1947; Lindenburg 2004; Lee and $\mathrm{Wu}$ 2013). So far, forces are generated also in the radial direction, which usually are neglected Velocity stream lines have been extracted from full rotor CFD computations carried out by Guntur (2013) on the MEXICO rotor blade. They found, for incoming wind speed corresponding to fully attached flow $U_{\infty}=12 \mathrm{~m} / \mathrm{s}$, that the centrifugal force is null and have no effect on the flow around the rotor blade. For incoming wind speed $U_{\infty}=15 \mathrm{~m} / \mathrm{s}$, the radial flow begins to appear at the separated region. Nevertheless, the flow is dominantly in the chord wise direction which means that the influence of centrifugal force is negligible. By increasing the incoming wind speed $U_{\infty}$ from $17 \mathrm{~m} / \mathrm{s}$ to $28 \mathrm{~m} / \mathrm{s}$, the radial flux increases causing the fluid particles to gather in the center of the blade airfoil (chord wise direction) and to form a fluid mass that travels radially. This is due to the influence of the centrifugal force that becomes dominant. Lee and $\mathrm{Wu}$ (2015) carried out a Tomographic Particle Image Velocimetry (Tomo-PIV) study on stall delay of HAWT rotor blade, they measured the radial velocity magnitude over the rotor blade at stall regime and they found that the radial velocity component is equal to zero on the near root region of the blade and increase radially to attain the maximum value at the near tip region. They described the radial forces acting on the flow field, causing the radial flow effect, as centrifugal pumping.

Going through the previous conclusions, a new Radial Flow Model (RFM) is presented here and is based on two main considerations: 
- $\quad$ The radial flow over the rotor blade increases with the incoming wind speed;

- consequently, the radial force on the rotor blade increases.

- The radial flow is negligible from the root to the midspan region of the rotor blade; as a result, the radial force influence is at the upper midspan region of the rotor blade.

Based on the above considerations, for a rotor blade with a rotation speed $\Omega$, the centrifugal force per unit volume of the blade length acting on an infinitesimal fluid mass traveling radially at a given span wise position $r$ could be expressed as follows:

$\mathrm{f}_{\mathrm{r}}=\frac{\mathrm{dF}_{\mathrm{r}}}{\mathrm{dv}}=\varepsilon(\lambda)\left(\frac{\mathrm{r}}{\mathrm{R}}\right)^{3} \rho \mathrm{r} \Omega^{2}$

Where $\varepsilon(\lambda)$ is a function of the Tip Speed Ratio TSR. Its expression will be inferred from CFD computations as is presented in Sec. 4.12.

\subsection{Filtered LES governing equations}

Predictions would be performed using Large Eddy Simulation (LES) for incompressible flow conditionsaround the HAWT, the yielding filtered continuity and Navier-Stokes equations are presented, respectively, as follows:

$\frac{\partial \overline{\mathrm{u}}_{\mathrm{i}}}{\partial \mathrm{x}_{\mathrm{i}}}=0$

$\frac{\partial \bar{u}_{i}}{\partial t}+\frac{\partial\left(\bar{u}_{i} \bar{u}_{j}\right)}{\partial x_{j}}=$

$-\frac{1}{\rho} \frac{\partial \bar{p}}{\partial x_{i}}+\frac{\partial}{\partial x_{j}}\left[\left(v+v_{S G S}\right)\left(\frac{\partial \bar{u}_{i}}{\partial x_{j}}+\frac{\partial \bar{u}_{j}}{\partial x_{i}}\right)\right]+f_{B F, i}$

$\overline{\mathrm{u}}_{\mathrm{i}}, \overline{\mathrm{p}}$ are respectively the filtered velocity and pressure, $v$ and $v_{S G S}$ are respectively the kinematic and eddy viscosity. $f_{B F, i}$ are the actuator disk body forces acting upon the flow field. In the present paper, the most widely used Smagorinsky (1963) Sub Grid Scale (SGS) model has been used to model the eddies smaller than the grid size. The algebraic model invoked for the evaluation of the eddy viscosity is presented as follows:

$$
\begin{aligned}
& v_{S G S}=\left(C_{S} \Delta\right)^{2}\left|\bar{S}_{i j}\right| \\
& \bar{S}_{i j}=\frac{1}{2}\left(\frac{\partial \bar{u}_{i}}{\partial \bar{x}_{j}}+\frac{\partial \bar{u}_{j}}{\partial \bar{x}_{i}}\right)
\end{aligned}
$$

$\left|\overline{S_{i j}}\right|$ is the magnitude of the strain-rate tensor defined by Eq. $17, \Delta$ is the filter width and is usually chosen twice the cell size, where $C_{s}$ denotes the $C_{s}$ constant which varies usually in a range of $[0.1-0.2]$. In the present study, the constant $C_{S}$ has been set to 0.1 .

\section{NUMERICAL METHOD}

The mathematical model presented in Sec. 2 as well as all the correction approaches are implemented in $3 \mathrm{D}$ in-house subroutine designated "AD $\backslash$ LGMD" developed with OpenFOAM code. OpenFOAM is an open source CFD tool written in oriented object $\mathrm{C}++$ which consists on collection of libraries and executables. The actuator disk developed class is based on the finite volume formulation of the NavierStokes equations in curvilinear coordinates where the rotor is modeled as forces computed explicitly before being inserted as momentum sink in the 3D NavierStokes equations. For the convective term, the linear upwind stabilized

transport scheme a second order difference scheme for spatial terms and the Crank-Nicolson method for the time discretization have been applied. The incompressible solver for unsteady flows PISO (Issa 1986) (Pressure Implicit solution by Split Operator method) has been adopted to solve sequentially the implicit equations.

The computational domain, shown by Fig. 5 , is cubic with edges length of $L_{x}=L_{y}=L_{z}=10 D$. It is composed of a cylindrical pseudo-domain upstream and downstream the rotor. Transformation from Cartesian to cylindrical coordinates is done using the "AD $\_L G M D "$ subroutine. (a)

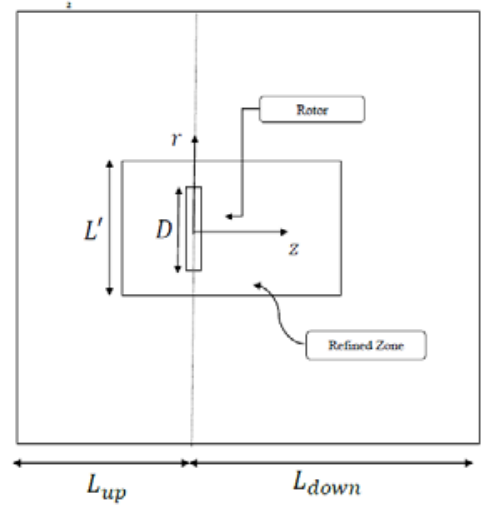

(b)

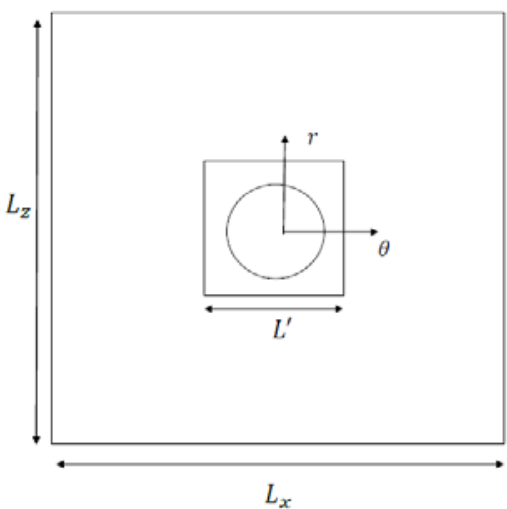

Fig. 5. Computational domain decomposition: (a) longitudinal view, (b) sectional view. 


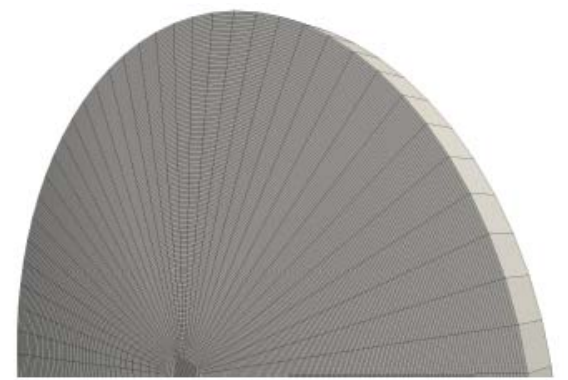

Fig. 6. Rotor O-type structured mesh on the computational domain.

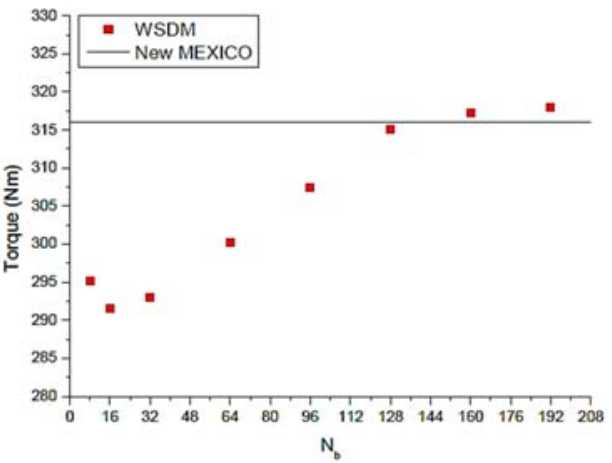

Fig. 7. Mesh sensitivity analysis on the optimum number $N_{b}$ of grid points along the rotor blade.

The computational domain is composed of a refined a regions upstream and downstream the rotor with an edge length of $L^{\prime}=2 D$. The inlet boundary condition is located at distance of $L_{u p}=4.5 \mathrm{D}$ from the rotor center, where the outlet is located at a distance of $L_{\text {down }}=5.5 D$.

A uniform velocity field of $U_{\infty}$ and a zero gradient of pressure have been prescribed at the inlet boundary condition where the outlet boundary conditions are set to zero gradient of velocity and a fixed pressure. The lateral boundary conditions are set as periodic.
The eddy viscosity based on Smagorinsky (1963) SGS model does not require boundary conditions.

The computational domain has been discretized throughout hexahedral structured mesh. The rotor and the refined domains upstream and downstream the rotor have been discretized with the O-type structured hexahedral mesh blocks, like shown by Fig. 6 , in order to make the mesh conform to the body surface. The rest of the computational domain has been discretized with a reasonable mesh size to ensure the numerical solution convergence. The mesh sensitivity study has been done through the MEXICO rotor for incoming wind speed of $U_{\infty}=$ $15 \mathrm{~m} / \mathrm{s}$ corresponding to an attached flow regimes. The MEXICO wind turbine is a three bladed $4.5 \mathrm{~m}$ diameter wind turbine with a rotational speed of 425 rpm. The MEXICO rotor blade is a variable chord and twist with a pitch angle of $-2.3^{\circ}$ and a coning angle $\gamma=0^{\circ}$. The blade is composed of three different aerodynamic profiles DU91-W2-250, RIS $\{\backslash \mathrm{O}\}-\mathrm{A} 1-21$ and NACA64-418 which are situated, respectively, from the root to the tip of the blade with transitions connecting the different aerodynamic profiles.

In order to determine an optimal mesh discritization during the ADM computations in the vicinity of HAWT rotor, a mesh dependency study for mainly the actuator points number and the mesh resolution upstream and downstream from the rotor is required. When looking at the predicted torque values variation versus the actuator points number, as shown by Fig. 7, it can be seen that the torque reaches a stable level for an actuator points number of $N_{b}=$ 128. It can be noticed that even if the actuator points number has been increased, the predicted torque is almost constant. As a results, an optimal actuator points number of $\mathrm{N}_{\mathrm{b}}=128$. has been obtained. In addition, high predictions accuracy on the near wake in vicinity of HAWT rotor is required; a mesh dependency study has been carried out upstream and downstream from the rotor. The predictions have been presented in Fig. 8 and Fig. 9 respectively. It can be seen that the predicted axial and radial velocity
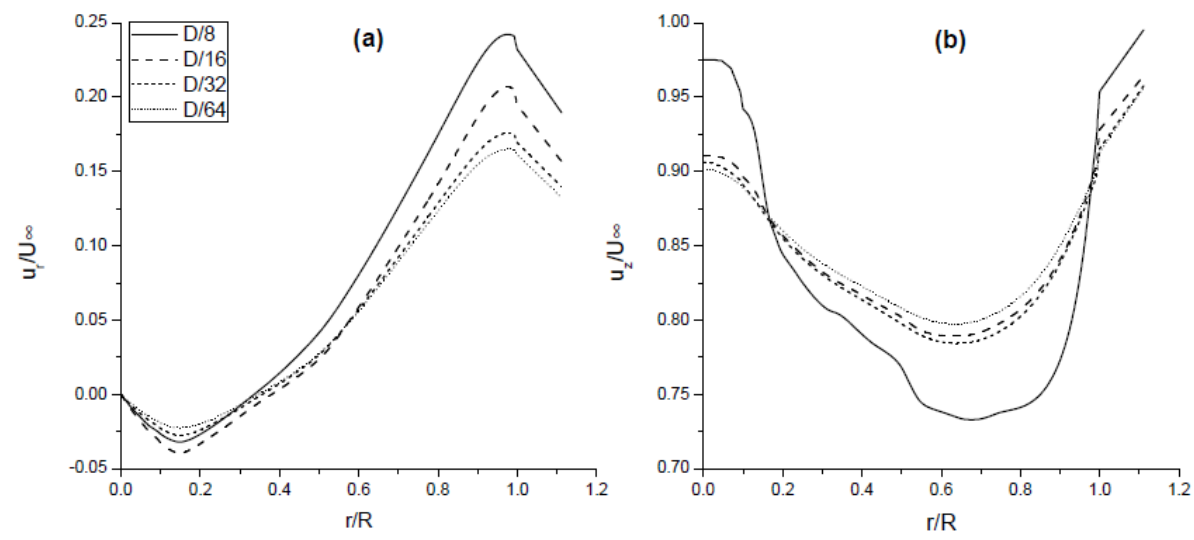

Fig. 8. Mesh sensitivity analysis on the radial distributions of velocity components $\left(U_{r}\right.$ and $\left.U_{z}\right)$ obtained at $0: 13 R$ upstream from the rotor. 
components distribution, at a distance of $0.13 \mathrm{R}$ upstream and downstream from the rotor, becomes negligible has been obtained for a mesh resolution of $\mathrm{D} / 64$ respectively. As a result, the mesh resolution of $\mathrm{D} / 64$, upstream and downstream from the rotor respectively, represents the optimum mesh size providing the best velocity distribution using minimum mesh requirements. The resulting discretized computational domain has been illustrated in Fig. 10.

4. CASE STUdy: Application OF THE NEW CORRECTION APPROACHES TO NEW MEXICO AND NREL PHASE VI DATA

In order to study the effect of rotation encountered on HAWTs, The NREL/NASA AMES Wind Tunnel Experiments (Simms et al. 2001; Fingersh et al. 2001) and the New MEXICO (Boorsma and Schepers 2016; Schepers et al. 2014) experiments provide large amounts of reference data to investigate the accuracy of the proposed numerical solutions on the performance predictions. Firstly, in this contribution, the numerical method will be validated with the New MEXICO rotor performances, normal and tangential sectional blade forces for an attached flow regimes corresponding to a tip speed ratios $\lambda=10$ and 6.67 respectively. Secondly, the new proposed stall delay model with new rotation correction approach called hereafter "ISDM-Rot" will be implemented, than coupled with the calibrated new radial flow model yielding on a combined model called hereafter "ISDM-Rot-Rf"; the obtained model will be compared with predictions of Du and Selig (1998) existing SDM; than validated with the New MEXICO measurements for separated flow regime corresponding to $\lambda=4.17$ and the NREL Phase VI lift coefficient distribution at two different radial positions corresponding to the near root region $(r / R=0.30)$, where the effect of rotation is significant, and a far root region $(r / R=$ 0.80 ) of the rotor blade; also, the predicted torque and thrust of the two wind turbines are discussed
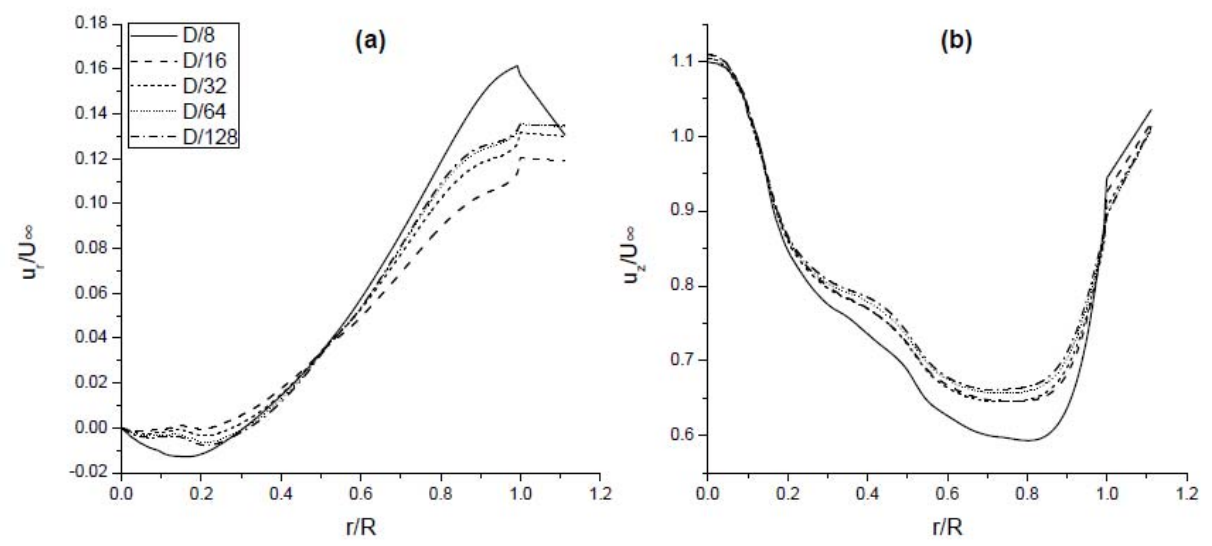

Fig. 9. Mesh sensitivity analysis on the radial distributions of velocity components $\left(U_{r}\right.$ and $\left.U_{z}\right)$ obtained at 0:13R downstream from the rotor.

(a)

(b)

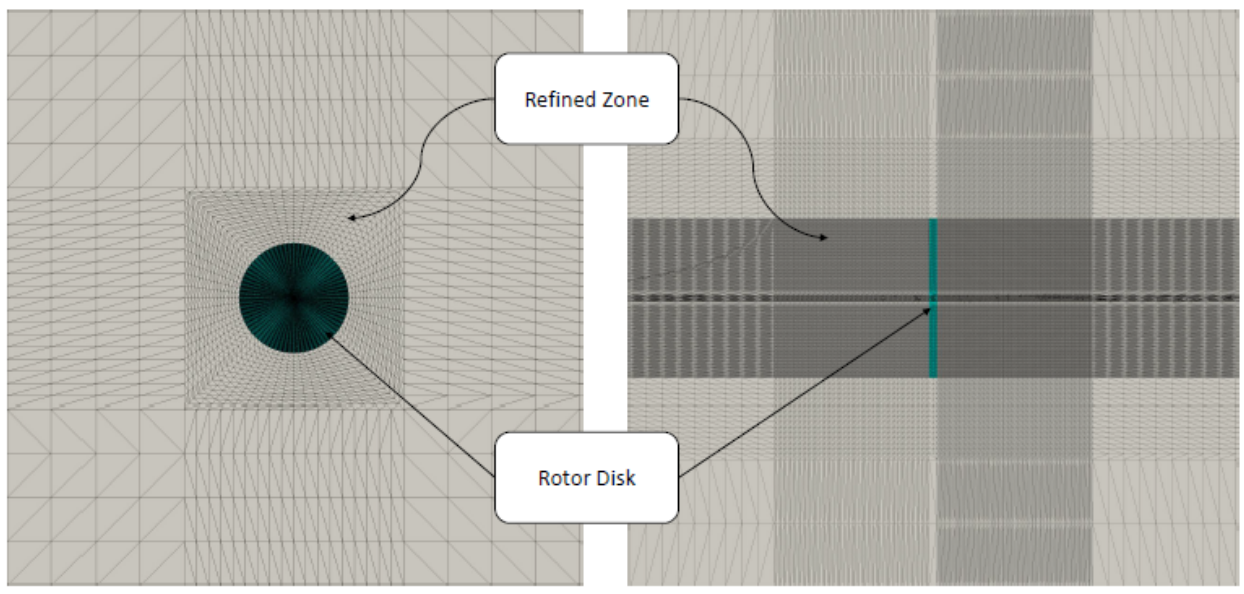

Fig. 10. Resulting discretized computational domain 

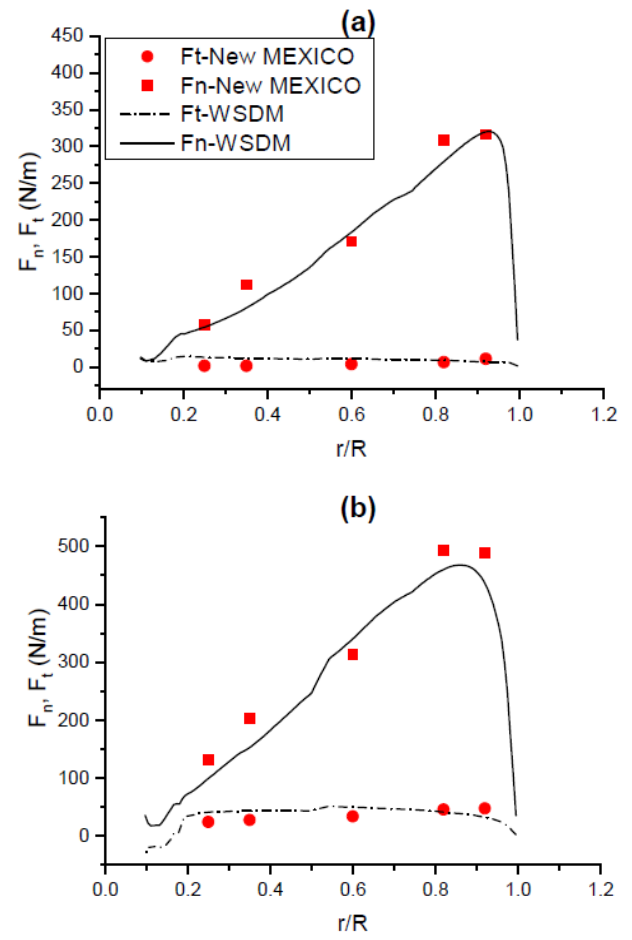

Fig. 11. Radial distributions of normal and tangential forces over the rotor blade, for TSR (a) $\mathrm{l}=\mathbf{1 0}$ and (b) $\mathrm{l}=\mathbf{6 : 6 7}$.

\subsection{New MEXICO rotor}

The proposed numerical method using the developed $3 \mathrm{D}$ subroutine "AD $\backslash$ LGMD" is validated first on the MEXICO wind turbine for the attached flow case.

\subsubsection{Attached flow regime}

Figure 11 shows the results of the normal and tangential forces distributions at two incoming wind speeds $\mathrm{U}_{\infty}$ of $10 \mathrm{~m} / \mathrm{s}$ and $15 \mathrm{~m} / \mathrm{s}$ corresponding to $\lambda=10$ and 6.67. As it can be seen, the predictions show good agreements in comparison to the measurements for both incoming wind speeds. On the other hand, Table 2 shows the corresponding torque and thrust predictions compared with those of experimental data for the same two incoming wind speeds. It has been thus recorded that for $U_{\infty}=10$ $\mathrm{m} / \mathrm{s}$, the torque and thrust shifts are respectively up to $7.5 \%$ and $0.2 \%$. While For $U_{\infty}=15 \mathrm{~m} / \mathrm{s}$, the torque and thrust shifts are up to, respectively, to $0.31 \%$ and
$2.5 \%$. These results confirm the capability of the proposed numerical method (AD $\backslash$ LGMD) to predict consistent results.

\subsubsection{Separated flow conditions}

The main objective of this paper is to study wind turbine rotors operating under deep stall regime, i.e. separated flows, where both the effect of rotation and the radial flow are significant, in order to determine its aerodynamic performances. Computations, using the proposed improved stall delay models (denoted ISDM-Rot and ISDM-Rot-Rf) have been carried out at deep stall regime (fully detached flow) corresponding to an incoming wind speed of $U_{\infty}=24$ $\mathrm{m} / \mathrm{s}$ (i.e. $\lambda=4.17$ ), compared with the existing SDM and with the New MEXICO measurements (Schepers et al. 2014; Boorsma and Schepers 2016).

Radial force rate estimation: For the separated flow conditions, the radial force (Eq. (13)) acting on the flow field for a given $T S R$ needs to be calculated. As explained above in Sec.2.23, the maximum value of the radial velocity component is attained at the tip of the rotor blade. As there are no measurements of the radial flow component over the rotor blade, it will be estimated based on the mean near wake radial velocity component, extracted from CFD computations, at a radial station downstream of the rotor at a distance of $0.13 R$. Figure 12 shows the mean near wake radial velocity component variation versus $\frac{r}{R}$, obtained at distance of $0.13 \mathrm{R}$ downstream the rotor for $\lambda=6.67$ and 4.17. As it can be seen in Fig. 12(a) for $\lambda=6.67$, the predicted radial component velocity WSDM is under-estimated compared with measurements; as it has been explained in Sec. 2.23, even though the flow is dominantly in the chord wise direction, the influence of centrifugal force is slightly present. In order to determine the amount of radial force rate, for $\lambda=$ 6.67 , computations for different values of $\varepsilon(\lambda)$ have been carried out where for a value of $\varepsilon(\lambda)=6 \%$ the radial velocity component has been corrected and fit well with the measured data as shown in Fig. 12(a). For the fully separated flow case corresponding to $\lambda=4.17$ as shown in Fig.12 (b), it can be noticed that the predicted radial velocity component WSDM, using the existing SDM and the new lift coefficient correction approach "ISDM-Rot" is significantly under-predicted compared to experimental measurements.

Table 2. Torque and thrust values for rotor blade, obtained at attached flow conditions $\lambda=10$ and 6.67: Comparison between predictions and experimental data.

\begin{tabular}{|c|c|c|c|c|}
\hline \multirow{2}{*}{} & \multicolumn{2}{|c|}{$\lambda=10$} & \multicolumn{2}{c|}{$\lambda=6.67$} \\
\cline { 2 - 5 } & $\mathrm{Q}(\mathrm{Nm})$ & $\mathrm{T}(\mathrm{N})$ & $\mathrm{Q}(\mathrm{Nm})$ & $\mathrm{T}(\mathrm{N})$ \\
\hline Exp & $68 \pm 2.4$ & $974 \pm 9.2$ & $316 \pm 2.5$ & $1663 \pm 15$ \\
WSDM & 73.57 & 976.06 & 315.02 & 1620.04 \\
Shift(\%) & 7.5 & 0.2 & 0.31 & 2.5 \\
\hline
\end{tabular}

WSDM: Without Stall Delay Model.

Q: Torque.

T: Thrust. 
On the other hand, the ISDM-Rot model provides slight improvements compared to the existing SDM, however, very high discrepancies are still recorded compared to the measured data due to the significant presence of the centrifugal force. In order to determine the amount of radial force rate, for $\lambda=$ 4.17, computations, for different values of $\varepsilon(\lambda)$, have been also performed where for a value of $\varepsilon(\lambda)=$ $16 \%$, the radial velocity component has been corrected and fit well with the measured data as shown in Fig.12.(b). Based on CFD computations and by assuming linear variation of $\varepsilon(\lambda)$ versus the TSR parameter which indicates how deeply separated is the flow over the HAWT blade, the proposed radial force rate has been defined as follows:

$\varepsilon(\lambda)=-0.04 \lambda+0.327$

Case study: The corresponding results of torque and thrust are presented in Table. 3 . As it can be seen, predictions without stall delay model corrections (WSDM) are consistently underestimated, due to appearance of the rotational effect. Important shift values have been noticed for both torque and thrust and are, respectively, equal to $35 \%$ and $12 \%$. To take into account the effect of rotation, the use of SDM has improved slightly the predictions, where the shift values of torque and thrust have been decreased, in comparison to those of WSDM case and are, respectively, equal to $25.93 \%$ and $10.7 \%$. By introducing the proposed model ISDM-Rot further improvements have been noticed on the resulting torque and thrust predictions; respectively, shift values up to $7.13 \%$ and $10 \%$ have been recorded. By the use of the new proposed model ISDM-Rot-Rf that takes into account of both effects: rotation and the radial flow over the wind turbine blade (ISDM-Rot$\mathrm{Rf}$ ), the precision of simulation results has been improved significantly; the torque and thrust shift values have been decreased to good uncertainty margin equal to, $1.5 \%$ and $10.5 \%$ respectively. The effect of rotation represents an increase of both the aerodynamic airfoil characteristics and the radial flow appearance due to the centrifugal pumping phenomenon overestimated on the near root region of the rotor blade, where for the near tip region, predictions are significantly under predicted.

Figure 13 shows the predicted normal and tangential forces distribution over the MEXICO rotor blade at deep stall regime for an incoming wind speed corresponding to $U_{\infty}=24 \mathrm{~m} / \mathrm{s}(\lambda=4.17)$. For the normal force distribution Fig. 13 (a), predictions WSDM and using SDM are significantly under predicted over the rotor blade. Nevertheless, By the introduction of the new proposed (ISDM-Rot-Rf), the predictions have been improved significantly and give good agreements with the measured data over
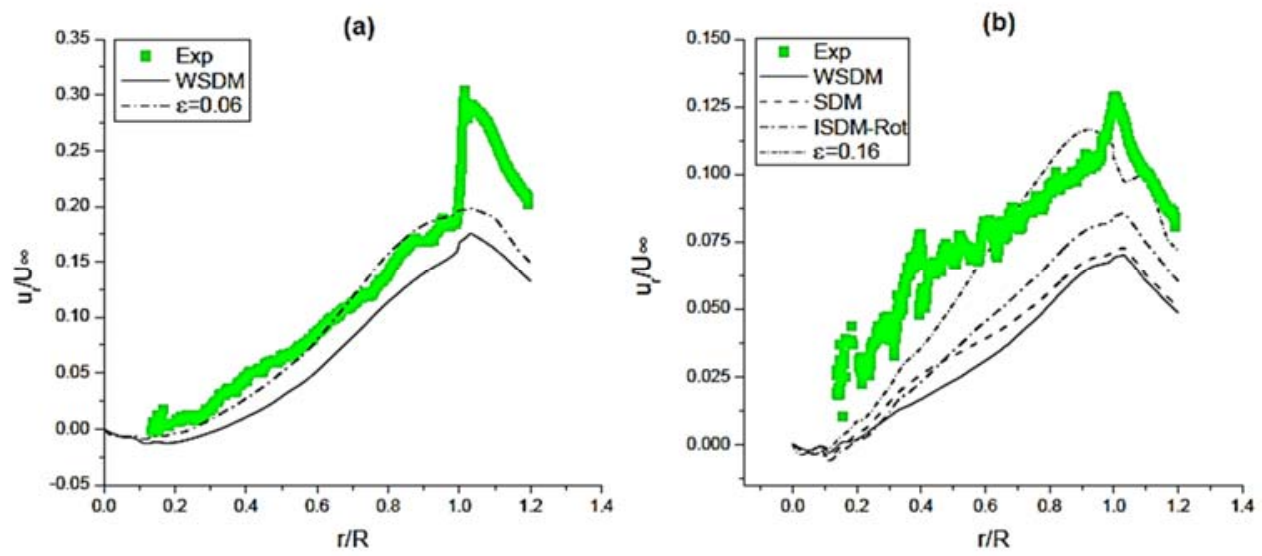

Fig. 12. Mean near wake radial velocity component variation versus $r R$ obtained at distance of 0:13R downstream from the rotor for (a) partially separated $\lambda=6.67(15 \mathrm{~m} / \mathrm{s})$ and (b) fully separated $\lambda=4.17$ $(24 \mathrm{~m} / \mathrm{s})$ flow regimes.

Table 3. Torque and thrust values for rotor blade, obtained at attached flow condition $\lambda=4.17$ : Comparison between predictions and experimental data.

\begin{tabular}{|c|c|c|c|c|c|c|c|c|c|}
\hline & Exp & WSDM & $\begin{array}{c}\text { Shift } \\
(\%)\end{array}$ & SDM & $\begin{array}{c}\text { Shift } \\
(\%)\end{array}$ & $\begin{array}{c}\text { ISDM- } \\
\text { Rot }\end{array}$ & $\begin{array}{c}\text { Shift } \\
(\%)\end{array}$ & $\begin{array}{c}\text { ISDM- } \\
\text { Rot-Rf }\end{array}$ & $\begin{array}{c}\text { Shift } \\
(\%)\end{array}$ \\
\hline$Q(\mathrm{Nm})$ & $715 \pm 2.0$ & 463.96 & 35.11 & 529.55 & 25.93 & 663.9 & 7.13 & 704.3 & 1.53 \\
$T(N)$ & $2172 \pm 10.9$ & 1915.29 & 11.8 & 1939.41 & 10.7 & 2388.66 & 10 & 2400 & 10.5 \\
\hline
\end{tabular}


the rotor blade except the near tip region where predictions are slightly over estimated with an acceptable tolerance. For the tangential force distribution Fig. 13(b), predictions WSDM are under estimated over the rotor blade. The SDM gives inaccurate corrections, the predicted forces are significantly over estimated on the near root region of the rotor blade where for the near tip region, predictions are significantly under predicted. Prediction of ISDM-Rot-Rf fits well and gives good agreement with the measurements over the rotor blade. The ISDM-Rot-Rf provides the most accurate predictions compared to the existing models.

\subsection{NREL Phase VI rotor}

\subsubsection{Lift coefficient distribution}

It has been shown in Sec.4.1 that despite the airfoil aerodynamic characteristics have been corrected to take into account the effect of rotation, the proposed approaches fail to model the radial flow over the rotor blade at separated flow conditions due to the centrifugal force appearance. The new proposed centrifugal force formulation has been calibrated then it has been proved that the effect of rotation is the combination of both stall delay effect in the near root region of the rotor blade and the radial flow effect appearance at the tip of the rotor blade throw the New MEXICO measurements. In the present study the accuracy of the new combined model "ISDM-Rot-Rf" would be investigated, compared with the existing SDM and validated with the NREL Phase VI wind turbine measurements. The NREL Phase VI is a $10 \mathrm{~m}$ diameter two bladed HAWT. It is a variable chord and twist wind turbine with a rotational speed of $71.63 \mathrm{rpm}$, a pitch angle of $3^{\circ}$ and it consists of $\mathrm{S} 809$ uniform aerodynamic profile. Computations have been performed for wind speeds range of $5-24 \mathrm{~m} / \mathrm{s}$ at steady and non-yawed conditions.

Figure 14 shows the ISDM-Rot-Rf predicted lift coefficient distribution versus the AoAs at two different radial stations corresponding, respectively, to near
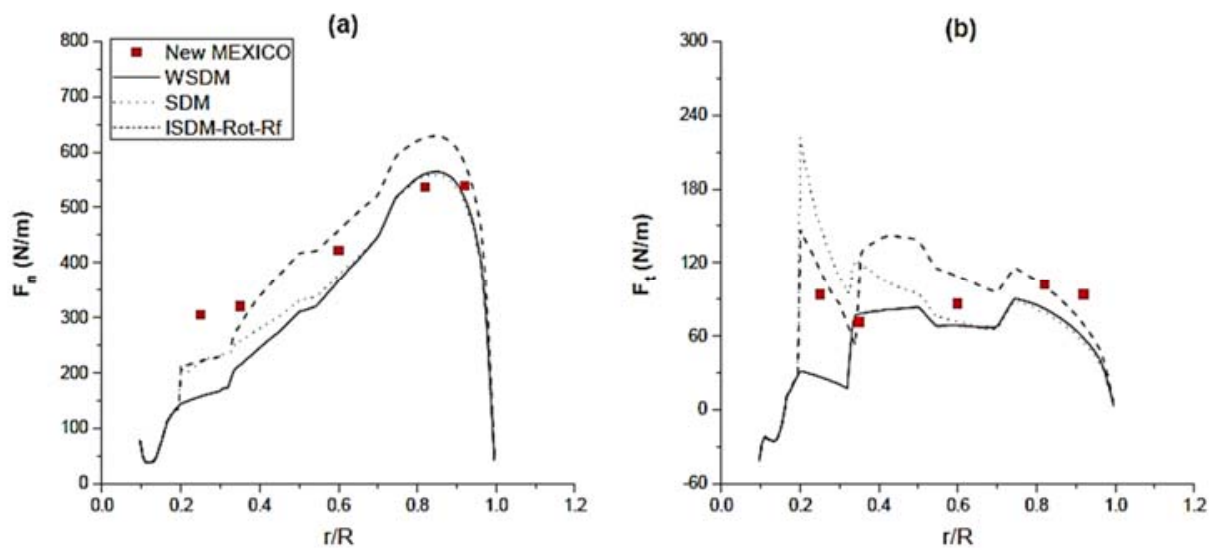

Fig. 13. Radial distributions of normal and tangential forces over rotor blade, obtained at $\lambda=4.17$ : (a)Normal force and (b) Tangential force.
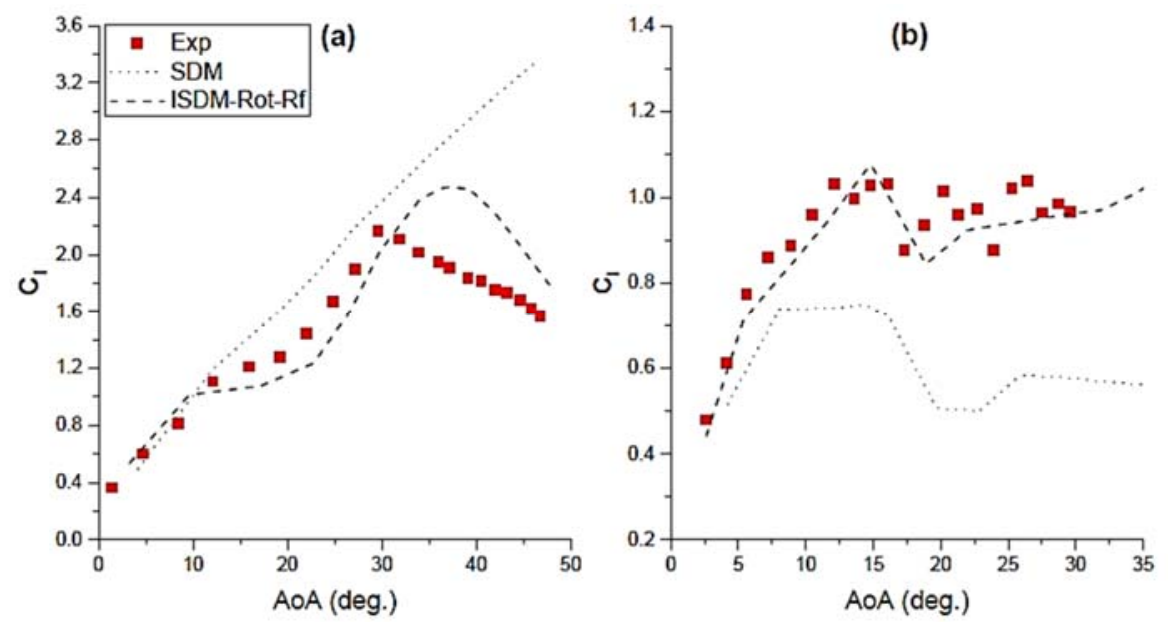

Fig. 14. Lift coefficient distribution at (a) $r / R=0.30$ and (b) $r / R=0.80$ radial stations of the NREL Phase VI rotor blade. 
$(\mathrm{r} / \mathrm{R}=0.30)$ and far $(\mathrm{r} / \mathrm{R}=0.80)$ root regions of the NREL Phase VI wind turbine blade. For the near root region $(\mathrm{r} / \mathrm{R}=0.30)$ shown by Fig.14(a), the effect of rotation is significant and it can be seen that the ISDM-Rot-Rf predictions give good agreement and a best realistic modeling of the effect of rotation compared to the SDM predictions. It can be seen that the ultimate limitation of the SDM corresponding on delaying stall to specific $\alpha_{s}$ and defining the appropriate rate of augmentation for each AoA has been taken into account using Eq. 12 where it can be noticed that the specific evolution of the lift coefficient is well modeled and the aerodynamic properties drop when stall occurs has been considered correctly. For the far root region $(\mathrm{r} / \mathrm{R}=0.80)$ presented by Fig.14(b), the effect of rotation decreased significantly where the measured lift coefficient is close to the $2 \mathrm{D}$ values with a slight increase. As it can be noticed, ISDM-Rot-Rf predictions are in a good agreements with the measured data compared with the SDM which gives under-estimated values. Most of SDM use the aspect ratio $(\mathrm{c} / \mathrm{r})$ to represent the sectional lift coefficient decrease over the rotor blade; however, HAWTs are high aspect ratio rotating machines which represent the second ultimate limitation causing performances being under estimated using SDM. Based on the lift coefficient evolution on the near and far root regions of the blade, it have been confirmed that SDM provides unphysical modeling of the effect of rotation.

\subsubsection{Torque and Thrust}

Figure 15 shows the ISDM-Rot-RF and SDM predicted mechanical torque and thrust through the NREL Phase VI wind turbine. For the NREL Phase VI wind turbine torque
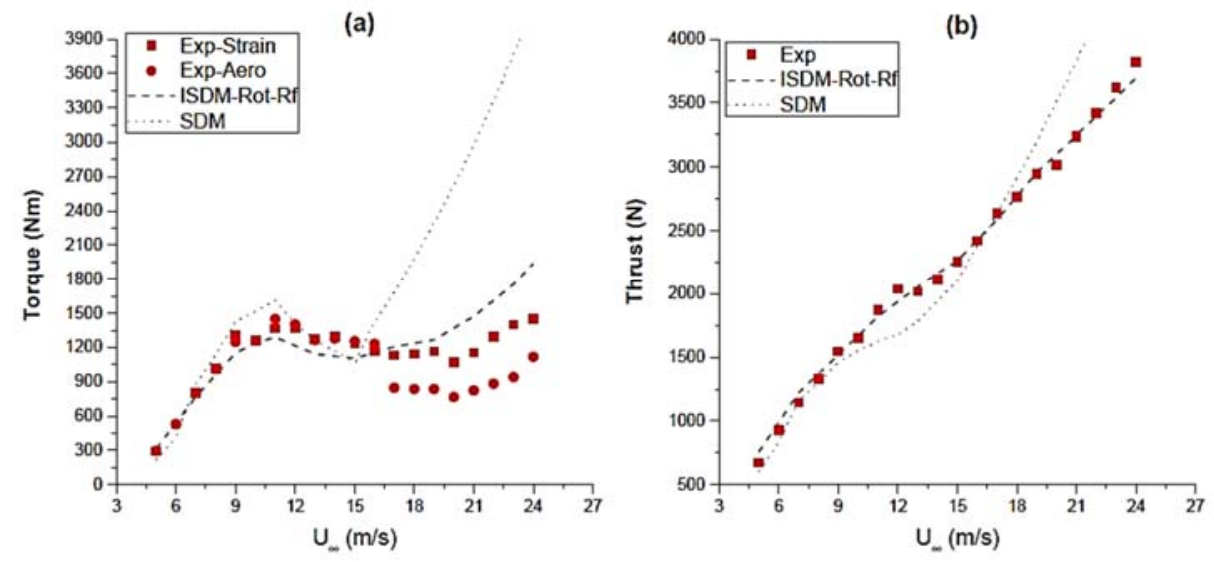

Fig. 15. Predicted (a) Torque and (b) Thrust of the NREL Phase VI rotor.

(Fig.15(a)), the obtained results can be divided into two parts of study which are, respectively, incoming wind speeds under $15 \mathrm{~m} / \mathrm{s}(\lambda=2.51)$ and incoming wind speeds upper than $15 \mathrm{~m} / \mathrm{s}(\lambda=2.51)$. It can be seen that for incoming wind speeds between $5 \mathrm{~m} / \mathrm{s}$ ( $\lambda=7.53)$ and $15 \mathrm{~m} / \mathrm{s}(\lambda=2.51)$, the predicted results of the ISDM-Rot-Rf and the existing stall delay models give good agreements with the measured rotor torque. However, for high incoming wind speeds between $15 \mathrm{~m} / \mathrm{s}(\lambda=2.51)$ and $24 \mathrm{~m} / \mathrm{s}(\lambda=$ 1.57), where stall occurs the most and have a significant effect, a remarkable over predictions using the SDM compared with the measured data is observed. Nevertheless, the predictions using the ISDM-Rot-Rf give noticeable improvement and encouraging agreements with the measured rotor torque. For the NREL Phase VI wind turbine thrust (Fig.15), the obtained results using SDM fails to predicted thrust for all incoming wind speeds, noticeable under-predictions have been observed for incoming wind speeds under $15 \mathrm{~m} / \mathrm{s}(\lambda=2.51)$ where significant over-predictions have been obtained for incoming wind speeds upper than $15 \mathrm{~m} / \mathrm{s}(\lambda=2.51)$. Nevertheless, predictions using the ISDM-Rot-Rf gives noticeable improvement and good agreement with the measured data for all incoming wind speeds. ISDM-Rot-Rf provides the best physical modeling of the effect of rotation compared to the existing models presented in the literature.

\section{CONCLUSIONS}

This paper presents an original correction approach, based on ADM calculations and CFD simulations, to take into account both effects of the blade rotational and the radial flow over the rotor blade to properly tune up the stall delay model, in order to improve the predictions of the HAWT aerodynamics performances. An in-house ADM subroutine, designated "AD LGMD" which has been developed with OpenFOAM code, combined with the 3D Navier-Stokes equations using LES based on Smagorinsky (1963) SGS model to describe the turbulent flow field around the HAWT rotor. New 
M. N. Hamlaoui et al. / JAFM, Vol. 15, No. 1, pp. 37-50, 2022.

MEXICO (Boorsma and Schepers 2016; Schepers et al. 2014) and NREL Phase VI (Simms et al. 2001;Fingersh et al. 2001) measurements have been used to investigate the validity of the CFD method. First, simulation results with the correction approach of the blade rotational (ISDM-Rot) alone being compared to those of a typical existing stall delay model (e.g. Du and Selig (1998)), have indicated some enhancement on performance predictions. Also, it has been proved that the radial flow phenomenon is independent of the effect of rotation, where stall delay correction approaches have been found unable to model the radial flux over the rotor blade. Then, in comparison with experimental data from New MEXICO, simulations results with both correction approaches, combined effects of the blade rotational and radial flow (ISDM-Rot-Rf) have demonstrated considerable improvements on performance predictions; namely, torque and thrust values showing very low discrepancies up to $1.0 \%$ and $10 \%$ respectively have been recorded. Then the combined model (ISDM-Rot-Rf) has been applied to the NREL Phase VI wind turbine where noticeable agreements for the lift coefficient distribution on the near and far root regions, mechanical torque and thrust have been obtained compared to the SDM and the measured data. From these simulation results, it could be drawn that flow around the rotor at stall regime is the combination of both, an increase of the aerodynamic airfoil characteristics due to the effect of rotation and the appeared centrifugal forces acting on the rotor blade.

\section{ACKNOWLEDGMENTS}

The authors are grateful to Universitée de Sherbrooke (Quebec, Canada) for providing to Mr. M. N. Hamlaoui, Ph.D. student, access to computational resources (beluga compute Canada), in order to perform the simulation calculations of this present work. Also, the support from DirectorateGeneral for Scientific Research and Technological Development (DG-RSDT) of Algerian government in the form of research grant to Prof. A. Smaili is gratefully acknowledged.

\section{REFERENCES}

Amini, S., M. R. Golzarian, E. Mahmoodi, A. Jeromin and M. H. Abbaspour-Fard (2021). Numerical simulation of the mexico wind turbine using the actuator disk model along with the $3 \mathrm{~d}$ correction of aerodynamic coefficients in openfoam. Renewable Energy 163, 2029 - 2036.

Boorsma, K. and J. Schepers (2016, sep). Rotor experiments in controlled conditions continued: New mexico. Journal of Physics: Conference Series 753, 022004.

Breton, S.-P., F. N. Coton and G. Moe (2008). A study on rotational effects and different stall delay models using a prescribed wake vortex scheme and nrel phase vi experiment data. Wind Energy 11(5), 459-482.
Chaviaropoulos, P. K. and M. O. L. Hansen (2000). Investigating Three-Dimensional and Rotational Effects on Wind Turbine Blades by Means of a Quasi-3D Navier-Stokes Solver. Journal of Fluids Engineering 122(2), 330-336.

Corrigan, J. J. and J. Schillings (1994). Empirical model for stall delay due to rotation. In American Helicopter Society Aeromechanics Specialists Conference, San Francisco, CA, Volume 21.

$\mathrm{Du}, \mathrm{Z}$. and M. Selig (1998). A 3-d stall-delay model for horizontal axis wind turbine performance prediction. In 1998 ASME Wind Energy Symposium, pp. 21.

Dumitrescu, H. and V. Cardos (2012). Inboard stall delay due to rotation. Journal of Aircraft 49(1), 101-107.

Dumitrescu, H., V. Cardos and A. Dumitrache (2007). Modelling of inboard stall delay due to rotation. In Journal of Physics: Conference Series, Volume 75, pp. 012022. IOP Publishing.

Fingersh, L., D. Simms, M. Hand, D. Jager, J. Cotrell, M. Robinson, S. Schreck and S. M. Larwood (2001). Wind tunnel testing of nrel's unsteady aerodynamics experiment. In 20th ASME Wind Energy Symposium.

Froude, R. E. (1889). On the part played in propulsion by differences of fluid pressure. Transactions of the Institution of Naval Architects 30, 390 .

Glauert, H. (1963). Airplane propellers, Aerodynamic theory. Dover Publications, New York.

Guntur, S. (2013). A detailed study of the rotational augmentation and dynamic stall phenomena for wind turbines. Ph. D. thesis, Technical University of Denmark.

Hansen, M. (2008). Aerodynamics of Wind Turbines: second edition (2 ed.). Earthscan.

Himmelskamp, H. (1947). Profile investigations on a rotating airscrew. Ministry of Aircraft Production.

Horlock, J. H. (1978). Actuator disk theorydiscontinuities in thermo-fluid dynamics. New York, McGraw-Hill International Book Co., 1978. $256 \mathrm{p}$.

Hu, D., O. Hua and Z. Du (2006). A study on stalldelay for horizontal axis wind turbine. Renewable Energy 31(6), 821 - 836 .

IRENA (2019). Future of wind: Deployment, investment, technology, grid integration and socio-economic aspects. In International Renewable Energy Agency.

Issa, R. I. (1986). Solution of the implicitly discretised fluid flow equations by operatorsplitting. Journal of Computational Physics 62(1), 40-65.

Lee, H. M. and Y. Wu (2013). An experimental study of stall delay on the blade of a horizontal-axis 
M. N. Hamlaoui et al. / JAFM, Vol. 15, No. 1, pp. 37-50, 2022.

wind turbine using tomographic particle image velocimetry. Journal of Wind Engineering and Industrial Aerodynamics 123, 56-68.

Lee, H. M. and Y. Wu (2015). A tomo-piv study of the effects of freestream turbulence on stall delay of the blade of a horizontalaxis wind turbine. Wind Energy 18(7), 1185- 1205.

Lindenburg, C. (2004). Modelling of rotational augmentation based on engineering considerations and measurements. In European Wind Energy Conference, London, 22-25.

Madsen, H. A. and F. Rasmussen (1988).Derivation of three-dimensional airfoil data on the basis of experiment and theory. In Proceedings Windpower(88), 166-174.

Masson, C. and A. Smaïli (2006). Numerical study of turbulent flow around a wind turbine nacelle. Wind Energy 9(3), 281-298.

Masson, C., A. Smaïli, I. Ammara and C. Leclerc (2000). Aerodynamic investigations on towershadow impacts for hawts. In Proceedings of the ASME Wind Energy Symposium, AIAA.

Masson, C., A. Smaïli and C. Leclerc (2001). Aerodynamic analysis of hawts operating in unsteady conditions. Wind Energy 4(1), 1- 22.

McCroskey, W. J. (1971). Measurements of boundary layer transition, separation and streamline direction on rotating blades, Volume 6321. National Aeronautics and Space Administration.

Prandtl, L. and A. Betz (1927). Vier abhandlungen zur hydrodynamik und aerodynamik. Kaiser Wilhelm-Institut für Strömungsforschung.

Rankine, W. J. M. (1865). On the mechanical principles of the action of propellers. Transactions of the Institution of Naval Architects 6.

Schepers, J., K. Boorsma and X. Munduate (2014). Final results from mexnext-i: Analysis of detailed aerodynamic measurements on a $4.5 \mathrm{~m}$ diameter rotor placed in the large german dutch wind tunnel dnw. In Journal of Physics: Conference Series, Volume 555, pp. 012089. IOP Publishing.

Shen, W. Z., R. Mikkelsen, J. N. Sørensen and C. Bak (2005). Tip loss corrections for wind turbine computations. Wind Energy 8(4), 457-475.

Simms, D., S. Schreck, M. Hand and L. J. Fingersh (2001). Nrel unsteady aerodynamics experiment in the nasa-ames wind tunnel: a comparison of predictions to measurements. Technical report, National Renewable Energy Lab., Golden, CO (US).

Smagorinsky, J. (1963). General circulation experiments with the primitive equations: I. the basic experiment. Monthly Weather Review 91(3), 99-164.

Smaïli, A. and C. Masson (2003, 01). Numerical Investigations of Nacelle Anemometry for Horizontal Axis Wind Turbines. In Wind Energy Symposium, 389-398.

Smaïli, A. and C. Masson (2004). On the rotor effects upon nacelle anemometry for wind turbines. Wind Engineering 28(6), 695-713.

Snel, H., R. Houwink, J. Bosschers (1994). Sectional prediction of lift coefficients on rotating wind turbine blades in stall. Netherlands Energy Research Foundation Petten, Netherlands.

Sørensen, J. N. and A. Myken (1992). Unsteady actuator disc model for horizontal axis wind turbines. Journal of Wind Engineering and Industrial Aerodynamics 39(1-3), 139-149.

Sørensen, J. N., K. Nilsson, S. Ivanell, H. Asmuth and R. F. Mikkelsen (2020). Analytical body forces in numerical actuator disc model of wind turbines. Renewable Energy 147, 2259 - 2271.

Stevens, R. J., L. A. Martínez-Tossas and C. Meneveau (2018). Comparison of wind farm large eddy simulations using actuator disk and actuator line models with wind tunnel experiments. Renewable Energy 116, 470 - 478.

Tahani, M. and M. Moradi (2016). Aerodynamic investigation of a wind turbine using cfd and modified bem methods. Journal of Applied Fluid Mechanics 9(1), 107-111.

Tata, M., A. Smaili and C. Masson (2018). Effect of grid topology on numerical simulations of flow fields around wind turbine nacelle anemometer. Journal of Applied Fluid Mechanics 11(6), 15691578.

Wimshurst, A. and R. H. J. Willden (2017). Analysis of a tip correction factor for horizontal axis turbines. Wind Energy 20(9), 1515-1528.

Yu, G., X. Shen, X. Zhu and Z. Du (2011). An insight into the separate flow and stall delay for hawt. Renewable Energy 36(1), 69 - 76. 